\title{
Face recognition of face images with hidden parts using gabor wavelets and PCA
}

\author{
Faouzia Ennaama ${ }^{1}$, Khalid Benhida ${ }^{1}$, Ahmed Boulahoual ${ }^{1}$ \\ ${ }^{1}$ Cady Ayyad University, High School of Technology, LAPSSII Laboratory, Safi-MOROCCO
}

\begin{abstract}
Face Recognition is one of the biometric methods that has recently gained significant attention at the level of research and the scientific community. However, in some special cases, face recognition methods can be sensitive to illumination, facial expressions, aging, face orientation, pose variation and hidden parts of face images, which make recognition very difficult. In this paper, we are interested in the hidden parts of a face image specifically those hidden by facial hair and/or a hair style. We first tested the Eigenfaces method for these modified images; but this approach failed to recognize them. So we have proposed an algorithm that combines Gabor magnitude and phase and PCA. To evaluate the efficiency of our algorithm, we used a variety of face images of FEI Brazilian database and hid the hairstyle and facial hair (barbs) to recognize them from the database images. The obtained results show that the proposed method (Gabor filter and PCA) attained high efficiency in the recognition for this type of problem.
\end{abstract}

\section{Corresponding Author:}

First Author,

Cady Ayyad University, High School of Technology,

LAPSSII Laboratory, Safi-MOROCCO,

Email: faouziaennaama@gmail.com

\section{Introduction}

Biometrics is defined by Pierson [1] as the determination of the identity of an individual by the mathematical analysis of its biological characteristics that can be either physiological (fingerprints, face, iris, contour of the hand,Finger vein, etc.) [2],[3], [4], or behavioral (signature, way of walking, etc.)[2].

What characterizes Biometrics is its universality [4],[5]. Moreover, they are measurable and unique. Theoretically that means two people cannot have exactly the same characteristics. They are also permanent [5]; in other words they do not vary over time.

Face recognition is one of the most widely used and appropriate biometric technologies [6],[7]. Seeing that, it is an activity that human beings perform naturally in their daily lives.

Several methods have been proposed to recognize a face in a $2 \mathrm{D}$ image and described in the literature, namely:

- Global (holistic) approaches [8], which use the entire face as the input data for the proposed recognition system. These data will be transformed as a vector, and then be projected onto a subspace of a small size.

- Local recognition approaches [8], which use some features or areas of the face to apply biometric analysis such as eye distance, nose length, chin shape, etc., as well as their positions and local statistics (geometric and / or appearance). 
- Hybrid approaches [8], which combine global and local characteristics in order to exploit the advantages of the two approaches. They use the local features of the global facial region to recognize it as the human perception system does. Hybrid approaches have the possibility to offer better performance than individual holistic or local methods, since more comprehensive information could be employed.

More than that, Face Recognition can be sensitive to pose variations, illumination, facial expression, age, occlusion, facial hair and hidden parts of face images (occlusion) [9]. These factors and others make face recognition very difficult and represent a big challenge for researchers to propose algorithms in order to handle, the above-mentioned problems. Among the methods that have shown a great success in face recognition we cite: Gabor Wavelets [10], [11]. Multiple approaches based on this method have been developed such as: Gabor-Fisher Classifier (GFC) [12], Gabor Wavelet Network (GWN) [13], Gabor-Fisher [14], etc. The characteristics of spatial frequency (scale), spatial localization, and orientation selectivity of the Gabor wavelets have been recognized as one of the best face recognition representations [9].

Besides, Principal Component Analysis (PCA) [15] is one of the most popular approaches. It is also known as Eigenfaces method [15]. This statistical technique is largely used for dimensionality reduction in face representation and recognition problems [16], [17].

In this paper, we are interested in the hidden parts of a face specifically those hidden by facial hair and/or a hair style and we propose the reliability of Gabor Wavelets and the dimensionality reduction of PCA to develop an algorithm that combines Gabor magnitude and phase and PCA.

This paper is organized as follows; in section 2 we explain the Gabor Wavelets. In section 3, we describe the PCA and the Eigenfaces approach. Section 4 gives the simulation results of the proposed method tested on the public FEI database. The conclusion and perspective works are given in last section.

\section{Gabor Wavelets}

Gabor Wavelets (also called Gabor Kernels or filters) are fundamental to the EBGM (Elastic Bunch Graph Matching) algorithm. They have proven to be the optimal basis to extract local features like Fourier transforms in several domains such as: orientation selectivity, spatial localization and optimally localized in the space and frequency. This holistic method is applied to specific locations on a face or to the whole face image.

In the spatial domain, each 2D Gabor wavelet consists of a planar sinusoid and can be represented by two components: real and imaginary components [6], [18]:

- Real component

$$
\mathrm{g}(\mathrm{z}, \lambda, \Theta, \varphi, \sigma, v)=\exp \left(\frac{\chi^{\prime^{2}+v^{2} \mathrm{y}^{\prime 2}}}{2 \sigma^{2}}\right) \cos \left(2 \pi \frac{\chi^{\prime}}{\lambda}+\varphi\right)
$$

- Imaginary component

$$
g(z, \lambda, \Theta, \varphi, \sigma, v)=\exp \left(\frac{x^{\prime^{2}+v^{2} y^{\prime}}}{2 \sigma^{2}}\right) \sin \left(2 \pi \frac{\chi^{\prime}}{\lambda}+\varphi\right)
$$

Where $\mathrm{x}^{\prime}=\mathrm{x} \cos \theta+\mathrm{y} \sin \theta$ and $\mathrm{y}^{\prime}=\mathrm{x} \sin \theta+\mathrm{y} \cos \theta$ and $\mathrm{x}$ and $\mathrm{y}$ denote the pixel coordinates in the image plan. Typically, Gabor wavelets can take different forms, using parameters that control orientation, frequency, phase, size and aspect ratio:

- $\Theta$ defines the Orientation of the wavelet. $\Theta \in\{0, \pi / 8,2 \pi / 8,3 \pi / 8,4 \pi / 8,5 \pi / 8,6 \pi / 8,7 \pi / 8\}$.

- $\lambda$ specifies the Wavelength of cosine wave or frequency of the wavelet. $\lambda \in\{4,4 \sqrt{2}, 8,8 \sqrt{2}, 16\}$.

- $\varphi$ indicates the Phase of the sinusoid. Generally, Gabor wavelets are either even or odd. The real part (even) of the wavelet corresponds to a cosine function and the imaginary part (odd) form corresponds to a sine function. i.e. $\varphi \in\{0, \pi / 2\}$.

- $\sigma$ defines the Radius of the Gaussian. i.e $\sigma=\lambda$.

- $\gamma$ denotes the Aspect ratio of the Gaussian.

These parameters offer eight orientations, five frequencies, and two phases for a total of eighty different wavelets (see Fig.1). 


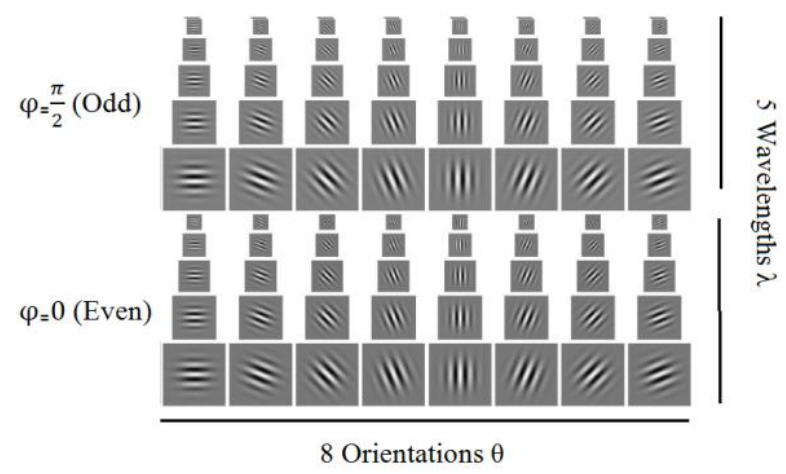

Figure1. Eighty different Gabor wavelets

\section{Principal Component Analysis}

Principal Component Analysis (PCA), which is also known as Karhunen-Loeve expansion [19], [20], is a highly useful technique for dimensionality reduction in compression and recognition problems. The method based on PCA technique is named Eigenfaces. It is proposed by Turk and Pentland in 1991. The main idea of Eigenfaces is to find a feature space with reduced dimension and then it is used for recognition.

Mathematically, a training set of facial images are collected and registered. Each image is reshaped into a vector, and a Principal Component Analysis (PCA) is performed on the training set. The eigenfaces are the eigenvectors [15] of the covariance matrix of the set of face images. Each face image in the training set can be represented exactly in terms of a linear combination of the eigenfaces. The number of possible eigenfaces is equal to the number of face images in the training set.

\subsection{Steps for Eigenfaces calculation}

Suppose a vector of dimension $\mathrm{N}^{2} \times 1$ corresponding to an $\mathrm{N} \times \mathrm{N}$ face image $\mathrm{I}(\mathrm{x}, \mathrm{y})$.

Obtain a set $\Gamma$ with $\mathrm{M}$ face images. Each image is transformed into a vector of size $\mathrm{N}^{2} \times 1$ and placed into the set $\Gamma=\{\Gamma 1, \Gamma 2, \ldots, \Gamma \mathrm{M}\}$ :

Compute the mean image $\Psi$ :

$$
\Gamma=\left(\begin{array}{cccc}
a_{1,1} & b_{1,1} & \cdots & z_{1,1} \\
\vdots & \vdots & & \vdots \\
a_{n, 1} & b_{n, 1} & \cdots & z_{n, 1} \\
\vdots & \vdots & \cdots & \vdots \\
a_{1, m} & b_{1, m} & \cdots & z_{1, m} \\
\vdots & \vdots & \cdots & \vdots \\
a_{n, m} & b_{n, m} & & z_{n, m}
\end{array}\right)
$$

$$
\Psi=\frac{1}{M} \sum_{i=1}^{M} \Gamma_{i}
$$

Find the difference $\Phi$ between the input image and the mean image:

$\begin{array}{ccc}\Phi \mathrm{i}=\Gamma \mathrm{i}-\Psi & \{\mathrm{i}=1,2, \ldots, \mathrm{M}\} \\ \text { Such as : } \quad \mathrm{A}=[\Phi 1, \Phi 2, \ldots, \quad \Phi \mathrm{M}] \quad\left(\mathrm{N}^{2} \times \mathrm{M} \text { matrix }\right)\end{array}$

Compute the covariance matrix $\mathrm{C}$ :

$$
\mathrm{C}=\frac{1}{\mathrm{M}} \sum_{\mathrm{i}=1}^{\mathrm{M}} \Phi_{\mathrm{i}} \Phi_{\mathrm{i}}^{\mathrm{T}}=\mathrm{AA}^{\mathrm{T}} \quad\left(\mathrm{N}^{2} \times \mathrm{N}^{2} \text { matrix }\right)
$$

Where $\mathrm{A}^{\mathrm{T}}$ the transpose matrix of $\mathrm{A}=[\Phi 1, \Phi 2, \ldots, \Phi \mathrm{M}]$

Compute the eigenvalues and the eigenvectors of the Covariance matrix $\mathrm{C}$ :

Considering the eigenvectors ei of $\mathrm{C}=\mathrm{A} \mathrm{A}{ }^{\mathrm{T}}$ associated to the eigenvalues $\lambda_{\mathrm{i}}$ :

$$
\mathrm{Ce} i=\lambda_{\mathrm{i}} \mathrm{e}_{\mathrm{i}}
$$


The eigenvectors $v_{i}$ of $L=A^{T} A$ associated to the eigenvalues $u_{i}$ are:

Such as: $\quad A^{\mathrm{T}} A v_{i}=u_{i} v_{i}$

$$
\mathrm{Lv}_{\mathrm{i}}=\mathrm{u}_{\mathrm{i}} \mathrm{v}_{\mathrm{i}}
$$

Since $\mathrm{C}=\mathrm{A} \mathrm{A}^{\mathrm{T}}$, the relation (10) becomes:

$$
\mathrm{C}\left(A v_{\mathrm{i}}\right)=\mathrm{u}_{\mathrm{i}}\left(\mathrm{Av}_{\mathrm{i}}\right)
$$

From (7) and (11) we notice that $A v_{i}$ and $u_{i}$ are respectively the eigenvectors $e_{i}$ and eigenvalues $\lambda_{i}$ of $C$ :

$$
\left\{\begin{array}{c}
e_{i}=A v_{i} \\
\lambda_{i}=u_{i}
\end{array}\right.
$$

Thus, to find the eigenvectors of $\mathrm{C}$, it suffices to compute the eigenvectors of $\mathrm{L}$ and multiply them by the matrix A.

The next step consists of selecting only the $\mathrm{K}$ best eigenvectors (Corresponding to the $\mathrm{K}$ largest eigenvalues. Each face $\Phi \mathrm{i}=\Gamma \mathrm{i}-\Psi$ in the training set can be represented as a linear combination of the best $\mathrm{K}$ eigenvectors:

$$
\omega_{\mathrm{K}}=\mathrm{e}_{\mathrm{k}}^{\mathrm{T}}\left(\Gamma_{\mathrm{i}-} \Psi\right)
$$

With $\mathrm{k}=1, \ldots, \mathrm{M}$ ' the number of $\mathrm{e}_{\mathrm{k}}^{\mathrm{T}}$ 's eigenvectors (Eigenfaces) only chosen.

The vectors $\omega_{\mathrm{K}}$ of each image are called "Weights" [15] and form a matrix named "Weight Vector":

$\Omega_{\mathrm{k}}^{\mathrm{T}}=\left[\omega_{1}, \omega_{2}, \ldots, \omega_{\mathrm{K}}\right]$.The K weights represent the contribution of each respective Eigenface. The vector $\Omega_{\mathrm{k}}^{\mathrm{T}}$, is taken as the "face-key" [15] for a face's image projected into Eigenspace. We compare any two "face-keys" by a simple Euclidean distance measure [15]:

$$
\varepsilon_{\mathrm{k}}^{2}=\left\|\Omega-\Omega_{\mathrm{k}}\right\|^{2}
$$

Where $\Omega_{\mathrm{k}}$ is a vector describing the Kth face class.

A face is classified as "known" when the minimum $\varepsilon_{\mathrm{k}}$ is below some chosen threshold $\theta_{\varepsilon}$. Otherwise the face is classified as "unknown" [15].

\section{Simulation Results}

In order to prove the importance of our approach, we recognize the test face image with hidden parts using Gabor Wavelets and PCA and compare the obtained results with those of Eigenfaces method.

\subsection{First test with Eigenfaces method}

Notwithstanding the fact that the Eigenfaces technique is very popular, easy and yields satisfying results in controlled circumstances; it has some limitations due to changes in lighting, angle, hidden parts of images (occlusion) and distance [9], [21].

We are interested in this article by the hidden parts of the face images, more precisely, those hidden by facial hair and/or a hair style (barbs). We tested this approach with a set of images where parts of the face were hidden.

We worked with the Brazilian FEI face database [22] .This database contains a set of face images taken between June 2005 and March 2006 at the Artificial Intelligence Laboratory of FEI in São Bernardo do Campo, São Paulo, Brazil. It consists of images captured from 200 individuals forming a total of 2800 images. Figure 2 shows some examples of image variations from the FEI face database.

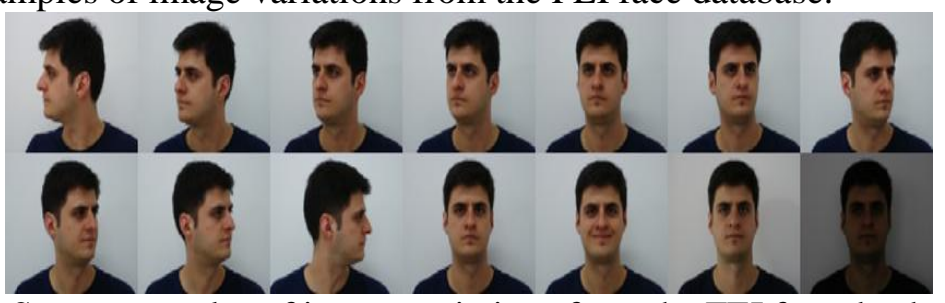

Figure 1.Some examples of image variations from the FEI face database [22].

For our study, we have selected 2 face images of 22 men and 8 women of different ages, for a total of 60 color images. In the first place, we have hidden the hair as well as other parts of a variety of faces (barbs for 
example) from all the images of the test database. The recognition is done by comparing the test image that we modified with all the images in the database using the Eigenfaces method.

The following results were obtained:
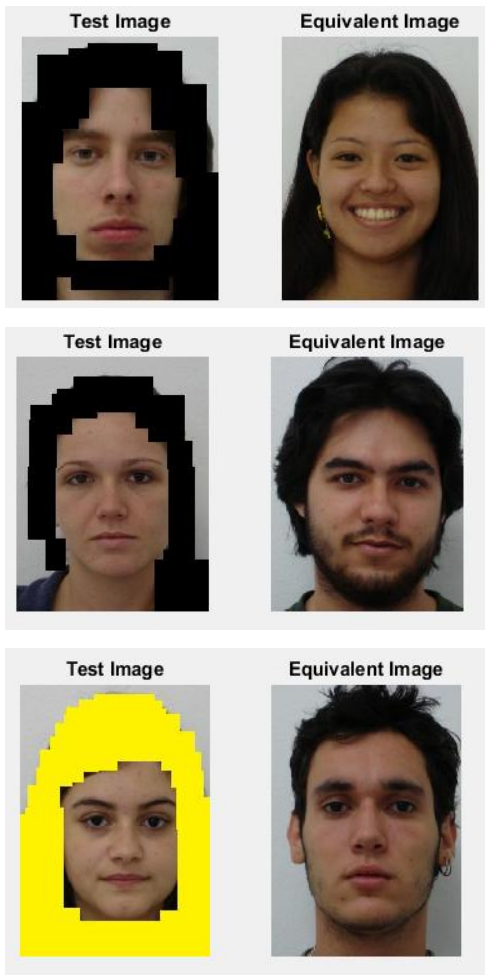

Figure 2. Some results of Eigenfaces method

The results of the first test show (see Fig.3) that the Eigenfaces method failed to recognize the hidden parts of face images, specifically, the parts hidden by facial hair and/or a hair style, and thence we can note that the Eigenfaces method is sensitive to the hidden parts of facial images.

\subsection{Second test with Gabor Wavelets and PCA}

To evaluate the feasibility and performance of the proposed algorithm Gabor Wavelets and PCA against the problem of hidden parts of face images, we worked with the same database used in the first test. The analysis steps of Gabor Wavelets and PCA algorithm for face recognition can be understood on the basis of the following steps:

Step 1: Convert each face image into grayscale image.

Step 2: Extract the Gabor magnitude and phase from each image of database as shown in figure 4. Thus, the face image is characterized by two components; magnitude and phase.

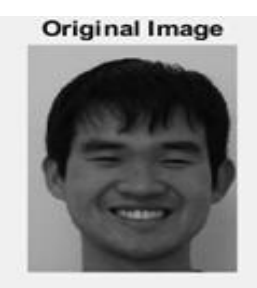

(a)

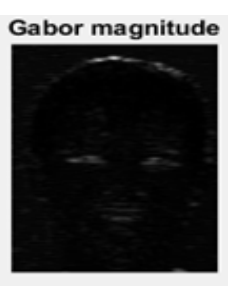

(b)

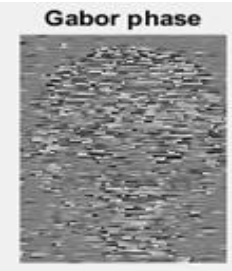

(c)

Figure 3. An example of extracting Gabor magnitude and phase. (a) Original face. (b) Gabor magnitude of the face. (c) Gabor phase of the face.

Step 3: Perform PCA for each Gabor magnitude and Gabor phase. Therefore, compute the eigenvalues and the eigenvectors of the Covariance matrix successively for each Gabor magnitude and phase, and obtain k Gabormagnitude-eigenspaces and k Gabor-phase-eigenspaces for every Gabor magnitude and every Gabor phase. 
Step 4: The k feature vectors obtained from k eigenspaces are merged into one vector called "Weight Vector" for all Gabor magnitude and phase.

Step 5: In the recognition phase, the test image with hidden facial hair and/or a hair style is projected onto the Gabor magnitude-eigenspace and Gabor phase-eigenspace after being converted into Gabor magnitude and phase and transformed to weight vector. The Euclidean distance is also exploited to find the distances between the test image and all the training images in Gabor-magnitude-subspace and Gabor-phase-subspace. Finally, the face image that has the minimum distance in Gabor magnitude and Gabor phase classified as "known" face if not "unknown".

Fig.5 shows some results obtained:

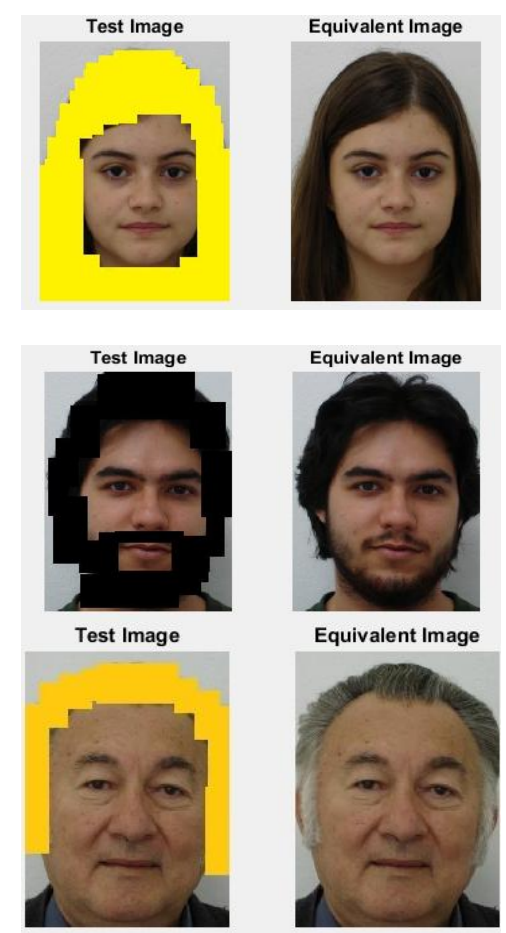

Figure 4. Some results obtained by our proposed approach.

From the results, it is clear that the proposed method is much more efficient, accurate and robust than the Eigenfaces method of the hidden parts of an image. Furthermore, it can identify and recognize easily all the face images of the database.

For comparison purposes, we can conclude that the proposed method Gabor Wavelets and PCA performs better than the Eigenface method.

\section{Conclusion}

In this paper, we proposed a robust algorithm that combines the magnitude and phase of Gabor to extract the characteristic vector and PCA for recognition. Subsequently, we tested the proposed algorithm and compared the obtained results with those of the Eigenfaces method. This algorithm has solved two types of problems of face recognition: the first is the hidden parts of images by the approach Gabor wavelets and the second is the reduction of dimensionality by the PCA method.

As a future work, we will expand the database. Therefore, the calculation becomes more complex. Accordingly, we propose to use parallel implementation to have a highest speed up in computation.

\section{References}

[1] J. PIERSON, "La biométrie: l'identification par le corps",Hermès Science Publ.: Lavoisier, 2007.

[2] A.K. JAIN, P. FLYNN and A.A. ROSS, (ed.). "Handbook of biometrics". Springer Science \& Business Media, 2007. 
[3] S.S. JUMAA and K. ZIDAN. "Finger vein recognition using two parallel enhancement ppproachs based fuzzy histogram equalization". Periodicals of Engineering and Natural Sciences, 2019, vol. 7, no 1, pp. 514-529.

[4] P.G. GAWANDE. "Quality-Dependent Fusion System using No-reference Image Quality Metrics for Multimodal Biometrics". Periodicals of Engineering and Natural Sciences (PEN), 2018, vol. 6, no 1, pp. 260-273.

[5] A.K. JAIN, A. ROSS and S. PRABHAKAR, "An introduction to biometric recognition". IEEE Transactions on circuits and systems for video technology, , 2004, vol. 14, no 1.

[6] C. LI, Y. HUANG, "Dependence Structure of Gabor Wavelets Based on Copula for Face Recognition". Expert Systems with Applications, 2019.

[7] M. WANG and W. DENG,"Deep face recognition: A survey". arXiv preprint arXiv:1804.06655, 2018.

[8] X. TAN, S. CHEN and Z.H. ZHOU, "Face recognition from a single image per person: A survey". Pattern recognition, 2006, vol. 39, no 9, pp. 1725-1745.

[9] S. A. KHAN, M. ISHTIAQ and M. NAZIR, "Face recognition under varying expressions and illumination using particle swarm optimization". Journal of computational science, 2018, vol. 28, pp. 94100.

[10] T.S. LEE, "Image representation using 2D Gabor wavelets". IEEE Transactions on pattern analysis and machine intelligence, 1996, vol. 18, no 10, pp. 959-971.

[11] A.C. PHAN, H.P. CAO and H.D. TRAN. "Face Recognition Using Gabor Wavelet in MapReduce and Spark". In : World Congress on Global Optimization. Springer, Cham, 2019. pp. 769-778.

[12] C. LIU and H. WECHSLER, "Gabor feature based classification using the enhanced fisher linear discriminant model for face recognition". IEEE Transactions on Image processing, 2002, vol. 11, no 4, pp. 467-476.

[13] V. KRÜGER and G. SOMMER, "Wavelet networks for face processing". JOSA A, 2002, vol. 19, no 6, pp. 1112-1119.

[14] Z. ELGARRAI, O. EL MESLOUHI and M. KARDOUCHI,"Offline Face Recognition System Based on Gabor-Fisher Descriptors and Hidden Markov Models". IJIMAI, 2016, vol. 4, no 1, pp. 11-14.

[15] M.A. Turk and A.P.Pentland, "Face recognition using eigenfaces". In : Proceedings. 1991 IEEE Computer Society Conference on Computer Vision and Pattern Recognition. IEEE, 1991. pp. 586-591.

[16] Y. P. SINGH andP. SINGH, "Face Recognition Using PCA Based Eigenfaces". MR International Journal of Engineering \& Technology, 2018, vol. 7, no 1, pp. 6-11.

[17] W.S. YAMBOR, B.A. DRAPER and J.R. BEVERIDGE, "Analyzing PCA-based face recognition algorithms: Eigenvector selection and distance measures". In : Empirical evaluation methods in computer vision. 2002. pp. 39-60.

[18] F.BELLAKHDHAR, K. LOUKIL and M. ABID, "Face recognition approach using Gabor Wavelets, PCA and SVM". International Journal of Computer Science Issues (IJCSI), 2013, vol. 10, no 2, p. 201.

[19] K. KARHUNEN, Über lineare Methoden in der Wahrscheinlichkeitsrechnung. Sana, 1947.

[20] M. LOČVE,"Fonctions Aléatoires de second order". Supplement to P. Levy, Processus Stochastiques et Mouvement Brownien. Gauthier-Villars: Paris, 1948.

[21] S. JAISWAL, "Comparison between face recognition algorithm-eigenfaces, fisherfaces and elastic bunch graph matching". Journal of global research in computer science, 2011, vol. 2, no 7, pp. 187-193.

[22] C.E. THOMAZ, "FEI face database". FEI Face DatabaseAvailable, 2012. 